\title{
Assimetria na Transmissão de Preços e Poder de Mercado: o caso do mercado varejista de etanol no estado de São Paulo
}

\author{
Jaqueline Zani dos Santos ${ }^{1}$, Danilo R. D. Aguiar² e \\ Adelson Martins Figueiredo ${ }^{3}$
}

Resumo: A presença de poder de mercado no varejo de combustíveis tem preocupado os brasileiros, motivando investigações dos órgãos antitruste e pesquisas econômicas. Entretanto, a carência de dados apropriados tem dificultado a estimação de indicadores diretos de poder de mercado, restando a possibilidade de se usar séries de preços. O objetivo deste estudo éidentificar a presença de poder de mercado no varejo de etanol em cidades de diferentes tamanhos. O método usado consiste em verificar se aumentos e reduções de preços são transmitidos assimetricamente aos consumidores, assumindo que transmissão mais intensa de aumentos de preços está relacionada à presença de poder de mercado. Foram utilizadas séries de preços mensais em níveis de distribuição e varejo para cidades de diferentes tamanhos do estado de São Paulo. Os resultados indicam a presença de poder de mercado, pois os varejistas conseguiram transmitir os aumentos de preço mais rapidamente e mais intensamente que os decréscimos na maioria das cidades. Tais resultados sugerem que a assimetria na transmissão de preços possa ser uma ferramenta analítica útil na investigação do poder de mercado e que as autoridades de defesa da concorrência devem ficar atentas à concentração e às condutas dos varejistas de combustíveis para proteger os consumidores.

Palavras-chaves: Poder de mercado; Transmissão de preços; Varejo; Etanol.

Abstract: The presence of market power in fuel retailing has been a major concern in Brazil, motivating antitrust investigations as well as economic research. However, lack of appropriate data precludes the estimation of straight market power indicators, leaving the

1. Mestre em Economia pela Universidade Federal de São Carlos (UFSCar). Sorocaba, SP, Brasil. E-mail: jaquezani@gmail.com

2. Professor Associado do Departamento de Economia da Universidade Federal de São Carlos (UFSCar). Sorocaba, SP, Brasil. E-mail: danilo@ufscar.br

3. Professor Adjunto do Departamento de Economia da Universidade Federal de São Carlos (UFSCar). Sorocaba, SP, Brasil. E-mail: adelson@ufscar.br 
possibility of using price series to identify market power. The objective of this study is to identify the presence of market power at the retail of fuel ethanol in cities of different sizes. The method adopted consists in verifying if price increases and decreases are transmitted asymmetrically to consumers, assuming that more intense transmission of price increases is related to the presence of market power. Monthly wholesale and retail price series of ethanol for cities with different sizes located in São Paulo State, Brazil, were used. The results indicate the presence of market power, since retailers were able to transmit price increases more rapidly and more intensively than price decreases for most cities. Such results suggest that asymmetry in price transmission can be a useful analytical tool to investigate market power and that antitrust authorities must observe the retail concentration and the behavior in the fuel market in order to protect consumers.

Key-words: Market power; Price transmission; Retail; Ethanol.

http://dx.doi.org/10.1590/1234-56781806-9479005302001

\section{Introdução}

Dados da Unica (2013) mostram que o crescimento da frota flex de automóveis, entre 2005 e 2012, passou de $6 \%$ para $57 \%$ da frota total, enquanto que a participação dos carros à gasolina diminuiu de $82 \%$ para $40 \%$. Tal tendência impulsionou o consumo de etanol hidratado no Brasil: como mostram os dados da ANP (2013), as vendas de etanol hidratado no país saíram de 4,6 milhões de $\mathrm{m}^{3}$, em 2000, para atingir um pico de 16,47 milhões de $\mathrm{m}^{3}$ em 2009, volume que sofreu retração em 2011 e 2012, quando atingiu 9,85 milhões de $\mathrm{m}^{3}$. A mesma base de dados mostra que, regionalmente, o destaque é o Sudeste, responsável por cerca de $70 \%$ do consumo nacional de etanol hidratado nos últimos dois anos. Em particular, destaca-se o estado de São Paulo, onde o preço do etanol é sistematicamente menor que nos outros estados da federação e onde normalmente esse combustível se mostra mais vantajoso do que a gasolina: apenas em 2011 o preço médio do etanol hidratado no estado de São Paulo superou a marca de $70 \%$ (atingiu 70,6\%) do preço médio da gasolina ${ }^{4}$, limite em que o consumo de gasolina se tornaria mais vantajoso do que o de etanol para a maioria dos carros flex.

4. Percentagem calculada pelos autores usando dados da ANP (2013).
A relevância do mercado de etanol hidratado para o Brasil e, principalmente, para o estado de São Paulo, evidenciada no parágrafo anterior, justifica preocupações com a possibilidade de ocorrerem perdas de bem-estar social no caso de as empresas da cadeia produtiva de etanol terem poder suficiente para fixar seus preços. Esta preocupação é maior em relação ao varejo, uma vez que os postos de combustíveis apresentam condições favoráveis à formação de cartéis, particularmente em cidades de menor porte (onde a concorrência é menor) ${ }^{5}$. Uma evidência da importância que tem sido dada a essa questão foi a publicação, por parte da Secretaria de Direito Econômico do Ministério da Justiça, da cartilha "Combate de Cartéis na Revenda de Combustíveis" que são relatadas as principais formas de exercício de poder de mercado no setor (SDE, 2009).

Portanto, para subsidiar a adoção de políticas que protejam os consumidores contra o abuso de poder econômico, é importante identificar se os postos de combustíveis do estado de São Paulo estão realmente exercendo poder de mercado na comercialização de etanol hidratado e verifi-

5. Martin (1993) apresenta algumas características que favorecem a formação de cartéis, as quais também estão presentes no varejo de combustíveis, tais como: elevada concentração de mercado, homogeneidade do produto e divulgação pública dos preços (o que permitiria identificar postos que não cooperassem com um cartel). 
car como isso varia em municípios de diferentes tamanhos.

Um problema que surge ao estudar o poder de mercado é a carência de dados que permitem estimar indicadores do grau de poder desfrutado pelas firmas no mercado. Como forma de lidar com essa questão, muitos trabalhos utilizam medidas de preços, tais como margens de comercialização, elasticidades de transmissão de preços, além do comportamento dos próprios preços, como indicadores de poder de mercado ${ }^{6}$. Outra forma de identificar a presença de poder de mercado é por meio da verificação da presença de assimetria na transmissão de preços (ATP). Conforme esclarecem Meyer e Von CramonTaubadel (2004), a ATP consiste em transmitir diferentemente acréscimos e decréscimos de preços, seja em velocidade, magnitude ou ambos. Para os mesmos autores, a ATP pode ter implicações em termos de bem-estar, pois um grupo não estaria se beneficiando da redução de preço (compradores) ou de seu aumento (vendedores). Em relação ao mercado brasileiro de combustíveis, Uchôa (2008) e Silva et al. (2010) estudaram a assimetria na transmissão de preços, sendo que o primeiro estudou apenas o mercado de gasolina e Silva et al., embora tenham incluído etanol em sua análise, concentraram-se nas relações entre os vários tipos de combustíveis e utilizaram o preço médio do etanol em nível nacional, não verificando variações em nível de município.

Dado esse panorama, o presente estudo tem como objetivo geral verificar se há exercício de poder de mercado por parte dos postos de combustíveis na revenda do etanol hidratado em uma série de municípios do estado de São Paulo, por meio da estimação de equações relacionando os preços dos distribuidores com os preços dos postos de combustíveis e do teste da assimetria na transmissão de preços. Especificamente, pretende-se: (a) determinar se os acréscimos e

6. Como exemplos de trabalhos que utilizam estes enfoques, ver Aguiar e Silva (2002), que estudaram o poder de mercado no varejo de carne bovina, e Carvalho e Aguiar (2005), que analisaram o poder de monopsônio da indústria de esmagamento de soja. decréscimos dos preços dos distribuidores são transmitidos com a mesma intensidade e com a mesma rapidez por parte dos postos de combustíveis; e (b) identificar se existe diferença no mecanismo de transmissão de preços entre municípios menores (onde a concorrência tende a ser menor) e maiores. Com isso, é possível se testar duas hipóteses, quais sejam: (i) se os varejistas conseguem exercer poder de mercado; e (ii) se o exercício de poder de mercado é mais provável nos municípios menores do que nos maiores.

Este artigo está organizado em cinco seções, incluindo esta introdução. Na seção 2 são apresentadas as considerações teóricas que embasam o trabalho, tanto em termos dos fatores que estão associados à capacidade de exercer poder de mercado quanto em relação ao conceito de assimetria na transmissão de preços. A seção 3 apresenta a natureza dos dados utilizados e os procedimentos empíricos adotados. Na seção 4 são apresentados e discutidos os resultados da pesquisa. A seção 5 conclui o trabalho.

\section{Referencial teórico}

\subsection{Condições favoráveis ao exercício de poder de mercado}

Poder de mercado, ou poder de monopólio, pode ser definido como a capacidade que as firmas possuem de manter seus preços acima do custo marginal e obter lucros supranormais (acima do lucro que ocorreria em competição perfeita) no longo prazo. De acordo com sua fonte, o poder de mercado pode ser de dois tipos: unilateral ou cooperativo ${ }^{7}$.

Conforme Aguiar (2000), o poder de mercado unilateral não dependeria das ações e das reações das empresas concorrentes, sendo intrínseco à firma ou às suas marcas. Uma firma teria poder unilateral quando existissem poucos substitutos, ou apenas substitutos inferiores, para seu produto. Sua origem estaria atrelada à diferen-

7. Ver Cotterill et al. (1996) e Aguiar (2000). 
ciação do produto ou à existência de barreiras à entrada de concorrentes. No setor de varejo de etanol, essas duas possibilidades estão presentes. Por um lado, a instalação de um novo posto de combustíveis tem elevados custos sunk e requer autorização por parte dos órgãos públicos, o que origina barreiras à entrada de concorrentes. Por outro, os postos de combustíveis, embora comercializem um produto que supostamente é não diferenciado, têm como diferenciar seus serviços por meio de localização, serviços de apoio, "bandeira" do distribuidor etc.

Em relação ao poder cooperativo, ainda segundo Aguiar (2000), o mesmo origina-se do processo em que as firmas agem coordenadamente para aumentar o lucro conjunto, seja por meio da redução da produção ou pelo aumento do preço (que seria o caso para os postos de combustíveis). A cooperação pode ser explícita, com a formação de um cartel (o que é ilegal no Brasil), ou implícita, em que as firmas sinalizam umas para outras, tentando mostrar suas ações na expectativa de que as outras ajam coerentemente para aumentar o lucro conjunto. O oposto da cooperação é a rivalidade, caracterizada por firmas agindo umas contra as outras.

Dentre os fatores que favorecem a cooperação entre firmas ${ }^{9}$, destacam-se a concentração do mercado, a homogeneidade do produto e a similaridade entre as estruturas de custo das firmas concorrentes, características que se mostram presentes no varejo de etanol, particularmente nos menores municípios. Além disso, outro fator considerado de grande valia para a cooperação, por permitir a identificação dos que não cooperam, é a divulgação pública dos preços de venda, fator este que também se mostra presente no setor.

Portanto, o setor varejista de combustíveis apresenta pré-condições para exercer poder de mercado, seja na forma unilateral, seja na forma

8. Ou seja, o investimento na implantação de um posto de combustíveis, por ser muito específico em termos do serviço prestado e da localização, causaria enormes perdas caso o investidor desejasse (ou precisasse) sair do negócio.

9. Ver Martin (1993) e Church e Ware (2000). cooperativa. Se este potencial está sendo efetivamente exercido é uma questão a ser identificada empiricamente.

\subsection{Assimetria na transmissão de preços e poder de mercado ${ }^{10}$}

A presença de transmissões assimétricas de preços tem sido identificada em diversos mercados, desde o início da década de 1980, mas as explicações teóricas para tal fenômeno ainda estão em seus primórdios (Ray et al., 2006). A maioria dos resultados tem indicado transmissão mais intensa de acréscimos de preços (ATP positiva) ou simetria ${ }^{11}$, havendo menor número de casos de transmissão mais intensa de decréscimos de preços (ATP negativa) ${ }^{12}$. Tem sido constatada ATP tanto em termos de magnitude (elasticidade de transmissão de acréscimos de preços maior do que a de decréscimos, ou vice-versa), quanto de velocidade (acréscimos de preços sendo transmitidos mais rapidamente do que decréscimos, ou vice-versa), assim como em ambas as formas, sendo que alguns estudos têm verificado ATP para o caso de pequenas mudanças de preços e simetria para o caso de grandes mudanças ${ }^{13}$.

As duas principais explanações para a ATP, segundo Meyer e Von Cramon-Taubadel (2004), seriam:

- Poder de mercado - este fator tem sido utilizado como justificativa para a ATP por diversos autores, sendo que a maioria sugere que o poder de mercado leva a ATP positiva. Apesar disso, tentativas de se testar empiricamente a relação entre poder de mercado e ATP, tal como fez Peltzman (2000), têm apresentado resultados conflitantes pela dificuldade de se encontrar

\footnotetext{
10. Por razões de espaço e foco do trabalho, este item apenas resume as principais questões ligadas à ATP, sem preocupação de citar e comentar os inúmeros trabalhos que têm sido feitos sobre o tema. Apenas alguns trabalhos são citados a título de ilustração. Para uma revisão profunda sobre o tema, ver Meyer e Von Cramon-Taubadel (2004).

11. Ver, por exemplo, Aguiar e Figueiredo (2011).

12. Ver Ward (1982).

13. Ver Levy et al. (2007).
} 
uma medida adequada para representar o poder de mercado. Mas como Meyer e Von Cramon-Taubadel (2004) argumentam, num contexto de oligopólio (e não monopólio) tanto ATP positiva quanto negativa seriam possíveis, dependendo da estrutura de mercado e da conduta das firmas.

- Custo de ajustamento de preços ${ }^{14}$ - caso haja custo de ajustamento de preço em algum estágio da cadeia produtiva, isto pode levar a ATP tanto neste como em outros estágios. Alguns exemplos: (a) Ward (1982) - o receio, por parte dos varejistas, de sofrerem prejuízos pelas perdas físicas (degradação) de produtos perecíveis que deixassem de ser vendidos favoreceria a ATP negativa; (b) Ray et al. (2006) - o custo de ajustamento de preço no varejo favoreceria ATP positivo do atacado; e (c) Levy et al. (2007) - o custo de busca de informação por parte de consumidores favoreceria ATP positiva dos varejistas.

Comparando esses dois motivos para a ATP, Meyer e Von Cramon-Taubadel (2004) argumentam que embora ambos possam produzir assimetria na velocidade de transmissão de preços, apenas o poder de mercado, aparentemente, seria capaz de levar um mercado à assimetria na transmissão de preços de longa duração. Com isso, a constatação de que um determinado estágio da cadeia produtiva consegue transmitir mais intensamente os acréscimos do que os decréscimos de preços, de forma duradoura, é um indício de exercício de poder de mercado por parte das firmas que atuam nesse estágio. Por outro lado, a constatação de que os decréscimos de preços são intensamente transmitidos é uma indicação de que as firmas estejam concorrendo mais agudamente entre si.

14. Este termo se refere à perda financeira que uma firma incorre caso mude o preço. Inclui custos explícitos, como é o caso de troca de etiquetas, reimpressão de anúncios etc., ou implícitos, tal como as perdas devido à redução das vendas em caso de aumento de preço.

\section{Metodologia}

\subsection{Modelo empírico}

A análise de assimetria implementada neste trabalho é a desenvolvida por Wolfram (1971) e aperfeiçoada por Houck (1977). Este procedimento tem como função identificar se acréscimos ou decréscimos de preços são transmitidos com a mesma intensidade. O procedimento, que será explicitado a seguir, consiste em se estimar regressões entre o preço do varejo e acréscimos e decréscimos do preço ao distribuidor, para cada uma das localidades estudadas (que serão discriminadas no próximo item). Para tal, lança-se mão de conceitos e técnicas da econometria de séries temporais ${ }^{15}$.

Houck (1977) considera um modelo em que a variável dependente, por exemplo, $P v$ (que representa o preço no varejo), é função apenas de uma variável independente, $\mathrm{Pa}$ (que representa o preço no atacado). Matematicamente, tem-se:

$$
\begin{aligned}
& \mathrm{DPv_{i }}=\mathrm{a}_{0}+\mathrm{a}_{1} \mathrm{DP} a_{i}^{a c}+\mathrm{a}_{2} \mathrm{D} P a_{i}^{d c}+\mathrm{e} \\
& \text { para } i=1,2, \ldots t
\end{aligned}
$$

Sendo que:

$$
\begin{aligned}
& \mathrm{D} P v_{i}=P v_{i}-P v_{i-1} \\
& \mathrm{D} P a_{i}^{a c}=P a_{i}-P a_{i-1} \text { se } P a_{i}>P a_{i-1} \\
& \mathrm{D} P a_{i}^{a c}=0 \text { se } P a_{i}<P a_{i-1} \\
& \mathrm{D} P a_{i}^{d c}=P a_{i-1}-P a_{i} \text { se } P a_{i}<P a_{i-1} \\
& \mathrm{DPa} a_{i}^{d c}=0 \text { se } P a_{i}>P a_{i}>P a_{i-1}
\end{aligned}
$$

Em que: $P v_{0}$ é o valor inicial de $P V ; P a_{0}$ é o valor inicial de $P a$.

$\mathrm{O}$ valor de $\mathrm{Pv}$ em que um ponto qualquer $t$ será:

$$
P v_{t}=P v_{0}+\sum_{i=1}^{T} D P v_{i} \quad \text { para } i=1,2,3 \ldots t, \ldots T
$$

15. Além das referências apresentadas neste texto que são específicas aos procedimentos e testes implementados, aos leitores interessados nos conceitos da econometria de séries temporais sugere-se consultar Hoffmann (2006), para um texto básico, ou Hamilton (1994), para uma leitura mais avançada. 
em que $T$ é o número total de observações, além do valor inicial. A diferença entre os valores corrente e inicial de Pv é a soma, de período a período, das mudanças que tem ocorrido. Assim,

$$
P v_{t}-P v_{0}=\sum_{i=1}^{T} \mathrm{D} P v_{i}
$$

substituindo a equação 1 na equação 3 e simplificando-a, tem-se

$$
P v_{t}-P v_{0}=a_{0} t+a_{1}\left(\sum D P a_{i}^{a c}\right)+a_{2}\left(\sum D P a_{i}^{d c}\right)
$$

Considerando-se $Y_{t}^{*}, Z_{t}^{*}$ e $W_{t}^{*}$ iguais a $\left(P V_{\mathrm{t}}\right.$ - $\left.P v_{0}\right),\left(\operatorname{SD} P a_{i}^{a c}\right)$ e $\left(\operatorname{SD} P a_{i}^{d c}\right)$, respectivamente, e incluindo o termo estocástico $\varepsilon_{\mathrm{t}}$, chega-se à equação a ser estimada:

$$
Y_{t}^{*}=a_{0 t}+a_{1} Z_{t}^{*}+a_{2} W_{t}^{*}+e
$$

Sendo $Z_{t}^{*}$ a soma de todos os acréscimos de preços, período a período, desde o valor inicial até o período $t$, e $W_{\mathrm{t}}^{*}$ é o similar para decréscimos de preços. A variável $Z_{\mathrm{t}}^{*}$ é sempre positiva, ao passo que $W_{\mathrm{t}}^{*}$ é sempre negativa. Se $\alpha_{0}$ não for zero, este pode ser considerado como um coeficiente de tendência na equação 5 . Se alguma outra variável afetasse $\Delta P v_{\text {i }}$ na equação 1 , esta também poderia ser incluída na equação 5 , como desvios de seus valores iniciais.

O teste de assimetria é feito testando-se a hipótese de que, em módulo, $\alpha_{1}$ é igual a $\alpha_{2}$. Caso se rejeite essa hipótese, há assimetria. Se $\left|\alpha_{1}\right|>$ $\left|\alpha_{2}\right|$, os acréscimos de preços são transmitidos mais intensamente que os decréscimos. Se $\left|\alpha_{1}\right|<$ $\left|\alpha_{2}\right|$, os decréscimos de preços são transmitidos mais intensamente. Vale ressaltar que no caso de os preços estarem na forma de logaritmos naturais, as elasticidades de transmissão serão os próprios coeficientes da equação estimada $\left(\alpha_{1}\right.$ e $\left.\alpha_{2}\right)$.

Havendo efeitos defasados, é possível se testar tanto a assimetria de curto prazo quanto a total. Os efeitos referidos como de curto-prazo são os efeitos ocorridos no mês corrente (sem defasagem), enquanto que os efeitos totais correspondem às somas dos efeitos de todos os meses considerados até o número máximo de defasagens significativas de cada equação de transmissão de preços. Se, por exemplo, houvesse defasagem de um mês, a especificação da expressão 5 a ser estimada seria:

$Y=a_{0}+a_{1}{ }^{\prime} D e c_{t}+a_{1}{ }^{\prime \prime} D e c_{t-1}+a_{2}{ }^{\prime} A c_{t}+a_{2} " A c_{t-1}+m_{t}(6)$

Dessa forma, o teste de assimetria de curto prazo (neste texto referido como "teste 1"), corresponderia a se testar se $\left|\alpha_{1}^{\prime}\right|=\left|\alpha_{2}^{\prime}\right|$ e o teste de assimetria total ("teste 2 ") corresponderia a se testar se $\left|\alpha_{1}^{\prime}+\alpha_{1}^{\prime \prime}\right|=\left|\alpha_{2}^{\prime}+\alpha_{2}^{\prime \prime}\right|$.

\subsection{Testes econométricos}

Após o deflacionamento, os preços foram convertidos em logaritmos neperianos e as séries foram submetidas a diversos testes econométricos para verificação de suas propriedades, sendo que após a estimação das equações de transmissão de preços entre os níveis de distribuição e varejo, para cada uma das cidades presentes na amostra, outros testes foram implementados para

Quadro 1. Procedimentos adotados para realização dos testes econométricos

\begin{tabular}{|l|l|}
\hline \multicolumn{1}{|c|}{ Tipo de teste } & \multicolumn{1}{c|}{ Procedimento } \\
\hline Raiz unitária & Teste NG e Perron (2001) \\
\hline Cointegração & teste de Johansen (1988) \\
\hline Número de defasagens & $\begin{array}{l}\text { 5 critérios foram analisados: LR (likelihood ratio); FPE (final prediction error); AIC (Akaike } \\
\text { information criterion); SC (Schwarz information criterion); e HQ (Hannan-Quinn information } \\
\text { criterion) - escolhendo-se o número de defasagens apontado pela maioria dos testes }\end{array}$ \\
\hline Autocorrelação entre os resíduos & Teste LM (Breusch-Godfrey) \\
\hline Heterocedasticidade & Teste de White \\
\hline Normalidade & Teste de Jarque-Bera \\
\hline
\end{tabular}

Fonte: Critérios dos autores. 
Quadro 2. Grupos, municípios selecionados por grupo e número de habitantes

\begin{tabular}{|c|c|c|}
\hline Grupo & Municípios & Número de habitantes* \\
\hline \multirow{3}{*}{$\begin{array}{c}\text { GRUPO1 } \\
\text { (menos de } 70.000 \text { habitantes) }\end{array}$} & Apiaí & 25.196 \\
\hline & Monte Alto & 46.647 \\
\hline & Paraguaçu Paulista & 42.281 \\
\hline \multirow{3}{*}{$\begin{array}{c}\text { GRUPO } 2 \\
\text { (de } 70.000 \text { a } 100.000 \text { habitantes) }\end{array}$} & Avaré & 82.935 \\
\hline & Itapeva & 87.765 \\
\hline & Jaboticabal & 71.667 \\
\hline \multirow{3}{*}{$\begin{array}{c}\text { GRUPO } 3 \\
\text { (de } 100.000 \text { a } 150.000 \text { habitantes) }\end{array}$} & Birigui & 108.722 \\
\hline & Botucatu & 127.370 \\
\hline & Guaratinguetá & 112.091 \\
\hline \multirow{3}{*}{$\begin{array}{c}\text { GRUPO } 4 \\
\text { (de } 150.000 \text { a } 250.000 \text { habitantes) }\end{array}$} & Marília & 216.684 \\
\hline & Presidente Prudente & 207.625 \\
\hline & São Carlos & 221.936 \\
\hline \multirow{3}{*}{$\begin{array}{c}\text { GRUPO } 5 \\
\text { (de } 250.000 \text { a } 400.000 \text { habitantes) }\end{array}$} & Franca & 318.785 \\
\hline & Limeira & 376.010 \\
\hline & Piracicaba & 364.872 \\
\hline \multirow{3}{*}{$\begin{array}{c}\text { GRUPO } 6 \\
\text { (de } 400.000 \text { a } 1.500 .000 \text { habitantes) }\end{array}$} & Campinas & 1.080 .999 \\
\hline & Osasco & 666.469 \\
\hline & Sorocaba & 586.311 \\
\hline
\end{tabular}

* Dados referentes ao Censo Demográfico de 2010 (IBGE, 2011).

Fonte: Critérios dos autores.

verificar a qualidade dos ajustamentos. Os testes realizados ${ }^{16}$ são apresentados no Quadro 1.

\subsection{Natureza dos dados}

Conforme já foi dito, a região escolhida para a análise foi o estado de São Paulo. Foram utilizadas séries de preços mensais, em nível de distribuição e de varejo (postos de combustíveis), coletados e divulgados pela ANP (ANP, 2011), referentes a 117 municípios desse estado. As séries de dados utilizadas compreendem o período de janeiro de 2005 a agosto de 2011. Todos os preços foram deflacionados utilizando-se o Índice de Preços ao Consumidor (IPC) da Fundação Instituto de Pesquisas Econômicas (FIPE, 2011).

Os municípios foram agrupados por tamanho populacional utilizando as informações do Censo Demográfico de 2010 do IBGE (IBGE, 2011), com o objetivo de se verificar a existência de diferenças no padrão de transmissão de pre-

16. As estimativas e os testes de hipóteses foram realizados no software EViews 6.0. ços entre municípios de diferentes portes. Os seis grupos, cujos limites estão discriminados no Quadro 2, envolvem municípios de até 1.500 .000 habitantes. O município de São Paulo foi excluído da pesquisa, tanto por sua população ser muito superior às dos demais municípios, o que causaria viés na análise caso esse fosse incluído em um dos grupos, quanto pela limitação da base de dados ${ }^{17}$. Dentro de cada grupo foram selecionados aleatoriamente três municípios ${ }^{18}$, conforme mostra o Quadro 2.

17. A base de dados da ANP não especifica a localização dos postos que fazem parte da amostra. No caso da cidade de São Paulo, isto se torna uma grande limitação, devido a sua heterogeneidade. Há dentro da cidade áreas de renda elevada, em que os consumidores seriam menos sensíveis aos preços e áreas de renda muito baixa; áreas de maior fluxo e áreas de menor fluxo de veículos; áreas com maior concentração de postos e áreas com menos postos; e assim por diante. Com isso, o preço médio da cidade de São Paulo seria uma variável pouco representativa.

18. Nota-se que os mercados individuais considerados nesta pesquisa (os municípios) não coincidem necessariamente com o tamanho do mercado relevante para a revenda de etanol considerado pelos órgãos de defesa da concorrência e pela ANP (200.000 habitantes). 


\section{Análise dos resultados}

Os resultados dos testes de raiz unitária de NG e Perron mostraram que, para todos os municípios, as séries de todas as variáveis eram estacionárias em nível (aos níveis de significância de $5 \%$ e 10\%), com exceção da soma dos acréscimos de preços. Excepcionalmente no município de Franca nenhuma variável se mostrou estacionária em nível. Essas séries, porém, se mostraram integradas de ordem um [I(1)]. Para estas séries I(1) foram realizados testes de cointegração de Johansen que mostraram, com base no teste do traço, ao nível de significância de 5\%, a existência de três vetores de cointegração para os municípios de Avaré, Botucatu, Itapeva, Jaboticabal, Marília, Paraguaçu Paulista e São Carlos; dois vetores de cointegração, ao nível de significância de $5 \%$, para o município de Apiaí; e um vetor de cointegração, ao nível de significância de 5\%, para os municípios de Birigui, Campinas, Franca, Guaratinguetá, Limeira, Monte Alto, Osasco, Piracicaba, Presidente Prudente e Sorocaba. Como todas as séries são estacionárias ou cointegradas, seguiu-se o procedimento adotado por Lass (2005) e por Aguiar \& Figueiredo (2011) para lidar com tais situações, de forma que as equações de transmissão de preços foram estimadas em nível.

Para a definição do número de defasagens significativas, dos critérios de escolha do número de defasagens, os que mais apresentaram o mesmo número de defasagens significativas foram: AIK (Akaike information criterion), SC (Schwarz information criterion) e HQ (Hannan-Quinn information criterion). Entretanto, o critério de Schwarz foi o mais observado na escolha por sua característica de impor uma penalidade maior pela inclusão de coeficientes adicionais a serem estimados. Para todos os municípios, os testes indicaram a utilização de uma defasagem, originando especificações semelhantes à expressão $6^{19}$.

19. Nos casos em que o coeficiente da variável defasada não se mostrou significativo, ao nível de $10 \%$ de significância pelo teste $t$ de Student, o modelo foi estimado sem a variável defasada.
Todas as equações foram estimadas pelo método de máxima verossimilhança, o que fez com que os resíduos assumissem distribuição normal, conforme se verificou por meio do teste de Jarque-Bera. Os resultados dos testes de White e Breusch-Godfrey permitiram rejeitar as hipóteses de presença de heterocedasticidade e autocorrelação entre os resíduos, respectivamente.

\subsection{Resultados para o Grupo 1}

No grupo em que se encontram os menores municípios, os resultados (Tabela 1) mostram um padrão, no curto prazo, de transmissão mais intensa de acréscimos de preços. Na soma dos períodos, os resultados diferem de acordo com o município. Supondo um aumento do preço do distribuidor da ordem de $10 \%$, os preços médios do varejo aumentariam, no mesmo mês, 6,15\%, 8,86\% e 7,25\%, respectivamente para Apiaí, Monte Alto e Paraguaçu Paulista. No caso de queda de $10 \%$ no preço do distribuidor, os preços do varejo diminuiriam, no mesmo mês, 2,43\%, 4,83\% e $5,09 \%$, respectivamente para Apiaí, Monte Alto e Paraguaçu Paulista. Portanto, o efeito instantâneo (ou de curto prazo), confirmado pelo teste 1 (Tabela 1), é de maior transmissão dos acréscimos de preços em todos os municípios.

Quando se leva em conta o efeito total (ou de longo prazo), os resultados diferem. Os resultados para os municípios de Apiaí e Monte Alto (considerando o nível de significância de 10\% para o segundo) mostram que, no total, os acréscimos de preços foram transmitidos mais intensamente, o que sugere que os varejistas teriam poder de mercado suficiente para evitar a transmissão de quedas de preços tanto no curto quanto no logo prazo. No caso de aumento de $10 \%$ no preço do distribuidor, o efeito total de aumento no preço do varejo seria de $7,24 \%$, em Apiaí, e 8,86\%, em Monte Alto, enquanto que uma redução de $10 \%$ no preço do distribuidor causaria reduções no preço do varejo de 5,74\%, em Apiaí, e 7,32\%, em Monte Alto. Em Paraguaçu Paulista, por outro lado, o efeito total reverte o efeito instantâneo. Um aumento de $10 \%$ no preço 
Tabela 1. Elasticidades de Acréscimos (Ac) e Decréscimos (Dec) de preços estimados por municípios do Grupo 1 (população menor que 70.000 habitantes)

\begin{tabular}{cccc}
\hline Variáveis e Testes & Apiaí & Monte Alto & Paraguaçu Paulista \\
\hline Constante & $-0,502^{*}(0,011)$ & $0,014^{* * *}(0,008)$ & $0,054^{*}(0,010)$ \\
$A c_{t}$ & $0,615^{*}(0,059)$ & $0,886^{*}(0,042)$ & $0,725^{*}(0,023)$ \\
$A c_{t-1}$ & $0,109^{* * *}(0,052)$ & - & - \\
Dec $_{t}$ & $-0,243^{*}(0,048)$ & $-0,483^{*}(0,037)$ & $-0,509^{*}(0,083)$ \\
Dec & $-0,331^{*}(0,043)$ & $-0,249^{*}(0,037)$ & $-0,267^{*}(0,078)$ \\
Teste 1 & $14,105^{*}[+]$ & $38,891^{*}[+]$ & $7,579^{*}[+]$ \\
Teste 2 & $12,005^{*}[+]$ & $4,956^{* *}[+]$ & $71,197^{*}[-]$ \\
\hline
\end{tabular}

Nota: Entre parênteses estão os valores do desvio padrão. * Significativo a 1\%.** Significativo a 10\%. Teste $1=$ Teste F de Assimetria Instantânea (no instante t). Teste 2 = Teste F de Assimetria Total (somando todos os períodos). Entre colchetes estão representados se os acréscimos [+] ou decréscimos [-] são transmitidos mais intensamente.

Fonte: Resultados da pesquisa.

do distribuidor continuaria causando aumento de $7,25 \%$ no preço do varejo (pois, neste caso, assim como para Monte Alto, não há efeito defasado), mas uma queda de $10 \%$ no preço do distribuidor originaria uma redução total de 7,76\% no preço do varejo. Esse resultado pode indicar que, nesse município, os varejistas não teriam como sustentar uma transmissão mais intensa de acréscimos de preços no longo prazo, possivelmente por alguma forma de rivalidade caracterizada pela redução de preço por parte de alguns postos que levariam os demais postos a transmitirem mais intensamente as quedas de preço no mês subsequente à variação de preço do distribuidor.

Além disso, nota-se que as elasticidades totais de transmissão de preços são menores do que a unidade, o que já era esperado. Como o produto vendido do varejo envolve custos de comercialização (salários, eletricidade etc.) que são mais estáveis do que o preço do etanol adquirido dos distribuidores, é natural que o preço no varejo varie menos do que em nível de distribuição ${ }^{20}$.

20. Ver Barros (1987).

\subsection{Resultados para o Grupo 2}

Para o Grupo 2 (Tabela 2), verifica-se que nos três municípios os acréscimos dos preços foram transmitidos pelos varejistas mais intensamente do que os decréscimos, tanto no curto prazo quanto no longo prazo, o que sugere que os varejistas dos três municípios desfrutam de poder suficiente para manter um padrão de aumento mais intenso dos acréscimos de preços. Em Avaré, um aumento de $10 \%$ no preço do distribuidor causaria aumento de $8,43 \%$ no preço do varejo no mesmo mês e não alteraria o preço do varejo no mês seguinte, enquanto que uma redução de $10 \%$ no preço do distribuidor provocaria redução instantânea de 4,6\% e uma redução total de 6,15\% no preço do varejo. Para Itapeva, um aumento de $10 \%$ no preço do distribuidor causaria aumento instantâneo de $5,42 \%$ e efeito total de $8,87 \%$, sendo que uma redução de $10 \%$ no preço do distribuidor causaria redução de 3,25\% no preço do varejo no mesmo mês e efeito total de 6,18\%. Em Jaboticabal, um acréscimo de 10\% no preço do distribuidor causaria um aumento de $7,55 \%$ no preço do varejo no mesmo mês, sendo este também o efeito total, enquanto que redução de $10 \%$ no preço do distribuidor causaria redução de 5,6\% no mesmo mês e redução total de 7,41\%. 
Tabela 2. Elasticidades de Acréscimos (Ac) e Decréscimos (Dec) de preços estimados por municípios do Grupo 2 (população entre 70.000 e 100.000 habitantes)

\begin{tabular}{cccc}
\hline Variáveis e Testes & Avaré & Itapeva & Jaboticabal \\
\hline Constante & $-0,033^{*}(0,010)$ & $-0,006(0,005)$ & $-0,034^{*}(0,005)$ \\
$A c_{t}$ & $0,843^{*}(0,044)$ & $0,542^{*}(0,000)$ & $0,755^{*}(0,011)$ \\
$A c_{\mathrm{t}-1}$ & - & $0,345^{*}(0,044)$ & - \\
Dec $_{\mathrm{t}}$ & $-0,460^{*}(0,066)$ & $-0,325^{*}(0,045)$ & $-0,560^{*}(0,046)$ \\
Dec & $-0,155^{*}(0,060)$ & $-0,293^{*}(0,057)$ & $-0,181^{*}(0,042)$ \\
Teste 1 & $21,281^{*}[+]$ & $23,163^{*}[+]$ & $20,456^{*}[+]$ \\
Teste 2 & $8,253^{* *}[+]$ & $12,445^{*}[+]$ & $13,573^{*}[+]$ \\
\hline
\end{tabular}

Nota: Entre parênteses estão os valores do desvio padrão. * Significativo a $1 \%{ }^{* *}$ Significativo a 5\%. Teste $1=$ Teste F de Assimetria Instantânea (no instante t). Teste 2 = Teste F de Assimetria Total (somando todos os períodos). Entre colchetes estão representados se os acréscimos [+] ou decréscimos [-] são transmitidos mais intensamente.

Fonte: Resultados da pesquisa.

\subsection{Resultados do Grupo 3}

No Grupo 3 (Tabela 3), mais uma vez, os acréscimos dos preços foram transmitidos pelos varejistas mais intensamente do que os decréscimos, no curto prazo, para todos os municípios. Na soma dos períodos, os municípios de Birigui e Botucatu apresentaram simetria, sugerindo que a diferença esteja na rapidez da resposta do setor varejista a acréscimos de preços. Em Guaratinguetá (considerando o nível de significância de 10\%), os acréscimos de preços continuaram sendo transmitidos mais intensamente, mesmo no longo prazo.

Analisando a partir de aumentos hipotéticos de $10 \%$ no preço do distribuidor: em Birigui, haveria aumento nos preços dos postos de $8,07 \%$, no mês corrente, e não haveria nenhum efeito no mês seguinte; em Botucatu, os preços nos pos- tos aumentariam 7,25\% no mês corrente e 8,36\% no total; e em Guaratinguetá, haveria aumento de 8,43\% apenas no mês corrente. Em caso de redução de $10 \%$ no preço ao distribuidor, haveria: redução instantânea de 4,78\% e redução total de 7,98\% no preço do varejo de Birigui; redução instantânea de 5,7\% e redução total de 7,53\% do preço ao varejo de Botucatu; e redução instantânea de $6,53 \%$ e redução total de $8,29 \%$ no preço dos varejistas de Guaratinguetá.

\subsection{Resultados do Grupo 4}

No Grupo 4 (Tabela 4), o município de Presidente Prudente se diferencia dos demais em todos os aspectos, pois no curto prazo esse município apresenta simetria na transmissão de preços, mas apresenta transmissão mais intensa dos decréscimos de preços na soma dos períodos.

Tabela 3. Elasticidades de Acréscimos (Ac) e Decréscimos (Dec) de preços estimados por municípios do Grupo 3 (População entre 100.000 e 150.000 habitantes)

\begin{tabular}{cccc}
\hline Variáveis e Testes & Birigui & Botucatu & Guaratinguetá \\
\hline Constante & $-0,084^{*}(0,010)$ & $-0,020^{* *}(0,010)$ & $-0,017^{*}(0,001)$ \\
$A c_{t}$ & $0,807^{*}(0,021)$ & $0,725^{*}(0,000)$ & $0,843^{*}(0,032)$ \\
$A c_{\mathrm{t}-1}$ & - & $0,111^{*}(0,035)$ & - \\
Dec & $-0,478^{*}(0,070)$ & $-0,570^{*}(0,042)$ & $-0,653^{*}(0,044)$ \\
Dec & $-0,320^{*}-(0,070)$ & $-0,183^{*}(0,046)$ & $-0,176^{*}(0,046)$ \\
Teste 1 & $21,253^{*}[+]$ & $13,069^{*}[+]$ & $16,990^{*}[+]$ \\
Teste 2 & 2,588 & 2,540 & $5,047^{* * *}[+]$ \\
\hline
\end{tabular}

Nota: Entre parênteses estão os valores do desvio padrão * Significativo a 1\%. *** Significativo a 10\%. Teste $1=$ Teste F de Assimetria Instantânea (no instante t). Teste 2 = Teste F de Assimetria Total (somando todos os períodos). Entre colchetes estão representados se os acréscimos [+] ou decréscimos [-] são transmitidos mais intensamente.

Fonte: Resultados da pesquisa. 
Tabela 4. Elasticidades de Acréscimos (Ac) e Decréscimos (Dec) de preços estimados por municípios do Grupo 4 (População entre 150.000 e 250.000 habitantes)

\begin{tabular}{cccc}
\hline Variáveis e Testes & Presidente Prudente & Marília & São Carlos \\
\hline Constante & $0,006(0,007)$ & $0,033^{*}(0,010)$ & $-0,011(0,014)$ \\
Ac $_{\mathrm{t}}$ & $0,645^{*}(0,033)$ & $0,912^{*}(0,033)$ & $0,861^{*}(0,047)$ \\
Dec $_{\mathrm{t}}$ & $-0,601^{*}(0,043)$ & $-0,548^{*}(0,046)$ & $-0,521^{*}(0,072)$ \\
Dec $_{\mathrm{t}-1}$ & $-0,318^{*}(0,047)$ & $-0,136^{*}(0,019)$ & $-0,136^{* * *}(0,071)$ \\
Teste 1 & 0,500 & $31,297^{*}[+]$ & $16,086^{*}[+]$ \\
Teste 2 & $14,790^{*}[-]$ & $10,314^{* *}[+]$ & $7,582^{* *}[+]$ \\
\hline
\end{tabular}

Nota: Entre parênteses estão os valores do desvio padrão. * Significativo a 1\%. ** Significativo a 5\%. Teste $1=$ Teste F de Assimetria Instantânea (no instante t). Teste 2 = Teste F de Assimetria Total (somando todos os períodos). Entre colchetes estão representados se os acréscimos [+] ou decréscimos [-] são transmitidos mais intensamente.

Fonte: Resultados da pesquisa.

Esse resultado sugere que fatores locais, que vão além do tamanho do município, possam ocasionar rivalidade entre os postos de combustíveis e, portanto, menor poder de mercado. Nos outros dois municípios, predominam as transmissões mais intensas de acréscimos de preços, tanto no curto prazo, quanto no longo prazo, padrão consistente com o que se verificou com o Grupo 3.

Para melhor dimensionar o tamanho dos impactos, pode-se, novamente, imaginar que haja variações de $10 \%$ no preço do distribuidor. No caso de um aumento de $10 \%$ no preço do distribuidor, o preço corrente do etanol no varejo aumenta 6,45\% em Presidente Prudente, 9,12\% em Marília e 8,61\% em São Carlos, não havendo efeito defasado em nenhum destes municípios. Se o preço do distribuidor diminuir $10 \%$, os preços nos postos de combustíveis sofreriam as seguintes variações: em Presidente Prudente, diminuição de 6,01\% no mês corrente e de 9,19\% no total; em Marília, diminuição de 5,48\% no mês corrente e de $6,84 \%$ no total; e em São Carlos, redução de $5,21 \%$ no mês corrente e de $6,57 \%$ no total.

\subsection{Resultados para o Grupo 5}

No Grupo 5 (Tabela 5), verifica-se que em todos os municípios os acréscimos de preços são transmitidos de forma mais intensa pelos postos de combustíveis no curto prazo, confirmando o padrão que predomina nos demais grupos. Já em relação ao efeito total, os 3 municípios apresentam comportamentos diferentes: em Franca, os decréscimos de preços são transmitidos mais intensamente; em Limeira, há simetria; e em Piracicaba, há transmissão mais intensa de acréscimos de preços. Mais uma vez, nota-se que fatores locais podem afetar o poder de mercado dos postos. Para Franca, os resultados indicam a existência de rivalidade que leva os postos a transmitirem mais intensamente os decréscimos de preços, embora num primeiro momento esses tenham conseguido transmitir mais intensamente as quedas de preços. Em Limeira, o poder de mercado se apresenta apenas na rapidez da resposta do setor varejista a acréscimos de preços, enquanto que em Piracicaba (considerando o nível de significância de 10\%) o poder dos varejistas tem permitido sustentar as transmissões mais intensas dos acréscimos de preços ao longo de todo o período de ajustamento.

Em termos do tamanho dos impactos, um aumento de $10 \%$ no preço do distribuidor acarreta aumentos, no mesmo mês, de 7,75\%, 8,97\% e 8,93\% nos preços do varejo de Franca, Limeira e Piracicaba, respectivamente, sendo nulos os efeitos defasados. Em caso de redução de $10 \%$ no preço do distribuidor, as reduções nos preços do varejo seriam: $6,21 \%$ no mês corrente e $7,96 \%$ no total, para Franca; 6,16\% no mês corrente e 8,98\% no total, para Limeira; e 6,75 no mês corrente e $8,87 \%$ no total, para Piracicaba. 
Tabela 5. Elasticidades de Acréscimos (Ac) e Decréscimos (Dec) de preços estimados por municípios do Grupo 5 (População entre 250.000 e 400.000 habitantes)

\begin{tabular}{cccc}
\hline Variáveis e Testes & Franca & Limeira & Piracicaba \\
\hline Constante & $-0,039^{*}(0,010)$ & $-0,017^{*}(0,004)$ & $-0,034^{*}(0,002)$ \\
Ac $_{\mathrm{t}}$ & $0,775^{*}(0,022)$ & $0,897^{*}(0,011)$ & $0,893^{*}(0,009)$ \\
Dec $_{\mathrm{t}}$ & $-0,621^{*}(0,053)$ & $-0,616^{*}(0,031)$ & $-0,675^{*}(0,011)$ \\
Dec $_{\mathrm{t}-1}$ & $-0,175^{*}(0,049)$ & $-0,282^{*}(0,039)$ & $-0,212^{*}(0,006)$ \\
Teste 1 & $9,362^{*}[+]$ & $49,092^{*}[+]$ & $1015,127^{*}[+]$ \\
Teste 2 & $13,643^{*}[-]$ & 0,102 & $4,196^{* * *}[+]$ \\
\hline
\end{tabular}

Nota: Entre parênteses estão os valores do desvio padrão. * Significativo a 1\%. ** Significativo a 10\%. Teste $1=$ Teste F de Assimetria Instantânea (no instante t). Teste 2 = Teste F de Assimetria Total (somando todos os períodos). Entre colchetes estão representados se os acréscimos [+] ou decréscimos [-] são transmitidos mais intensamente.

Fonte: Resultados da pesquisa.

\subsection{Resultados para o Grupo 6}

No Grupo 6 (Tabela 6), todos os municípios voltaram a transmitir mais intensamente os acréscimos de preços no curto prazo. Na soma dos períodos, Sorocaba e Osasco (considerando o nível de significância de 10\%) também apresentaram assimetria, onde os acréscimos de preços em nível de distribuidor foram transmitidos de forma mais intensa pelos varejistas. Mas em Campinas, que é justamente o maior município entre os três, a possível rivalidade entre os postos impediu que, no total, os acréscimos fossem transmitidos mais intensamente; o que se nota para esse município é que os decréscimos de preços acabam sendo transmitidos mais intensamente, embora no curto prazo os varejistas consigam exercer poder de mercado.

Completando a análise, conforme foi feito para os outros grupos, verifica-se que um aumento de $10 \%$ no preço do distribuidor causaria aumentos de $8,44 \%, 9,2 \%$ e $8,27 \%$ nos preços ao varejo de Sorocaba, Campinas e Osasco, respectivamente, sendo nulos os efeitos defasados. Se o preço diminuísse $10 \%$ em nível de distribuidor, os preços no varejo sofreriam reduções de: $5,25 \%$ no mesmo mês e $8,37 \%$ no total, em Sorocaba; $8,09 \%$ no mês corrente e $9,72 \%$ no total, em Campinas; e 6,04\% no mesmo mês e 8,2\% no total, para Osasco.

\subsection{Análise geral dos resultados}

O Quadro 3 apresenta um resumo dos resultados referentes à análise da assimetria na transmissão de preços no curto e no longo prazo. Os valores foram omitidos para permitir melhor visualização dos resultados. Nota-se que no curto prazo os acréscimos de preços são transmitidos

Tabela 6. Elasticidades de Acréscimos (Ac) e Decréscimos (Dec) de preços estimados por municípios do Grupo 6 (População entre 400.000 e 1.500 .000 habitantes)

\begin{tabular}{cccc}
\hline Variáveis e Testes & Sorocaba & Campinas & Osasco \\
\hline Constante & $-0,066^{*}(0,004)$ & $-0,027^{*}(0,005)$ & $-0,012^{*}(0,001)$ \\
Ac $_{\mathrm{t}}$ & $0,844^{*}(0,012)$ & $0,920^{*}(0,017)$ & $0,827^{*}(0,021)$ \\
Dec $_{\mathrm{t}}$ & $-0,525^{*}(0,025)$ & $-0,809^{*}(0,049)$ & $-0,604^{*}(0,033)$ \\
Dec $\mathrm{t}_{-1}$ & $-0,312^{*}(0,022)$ & $-0,163^{*}(0,045)$ & $-0,216^{*}(0,034)$ \\
Teste 1 & $226,380^{*}[+]$ & $6,083^{* *}[+]$ & $40,823^{*}[+]$ \\
Teste 2 & $6,634^{* *}[+]$ & $158,360^{*}[-]$ & $4,177^{* * *}[+]$ \\
\hline
\end{tabular}

Nota: Entre parênteses estão os valores do desvio padrão. ${ }^{*}$ Significativo a $1 \%$. ** Significativo a $5 \%$. ** Significativo a $10 \%$. Teste $1=$ Teste $\mathrm{F}$ de Assimetria Instantânea (no instante $\mathrm{t}$ ). Teste 2 = Teste F de Assimetria Total (somando todos os períodos). Entre colchetes estão representados se os acréscimos [+] ou decréscimos [-] são transmitidos mais intensamente.

Fonte: Resultados da pesquisa. 
Quadro 3. Padrão de transmissão de preços dos municípios (com significância de até 10\%)

\begin{tabular}{|c|c|c|c|}
\hline Grupo & Municípios & Curto Prazo (teste 1) & Total (teste 2) \\
\hline \multirow{3}{*}{ GRUPO1 } & Apiaí & $\mathrm{AC}$ & $\mathrm{AC}$ \\
\hline & Monte Alto & $\mathrm{AC}$ & $\mathrm{AC}$ \\
\hline & Paraguaçu Paulista & $\mathrm{AC}$ & DEC \\
\hline \multirow{3}{*}{ GRUPO 2} & Avaré & $\mathrm{AC}$ & $\mathrm{AC}$ \\
\hline & Itapeva & $\mathrm{AC}$ & $\mathrm{AC}$ \\
\hline & Jaboticabal & $\mathrm{AC}$ & $\mathrm{AC}$ \\
\hline \multirow{3}{*}{ GRUPO 3} & Birigui & $\mathrm{AC}$ & SIMÉTRICA \\
\hline & Botucatu & $\mathrm{AC}$ & SIMÉTRICA \\
\hline & Guaratinguetá & $\mathrm{AC}$ & $\mathrm{AC}$ \\
\hline \multirow{3}{*}{ GRUPO 4} & Marília & $\mathrm{AC}$ & $\mathrm{AC}$ \\
\hline & Presidente Prudente & SIMÉTRICA & DEC \\
\hline & São Carlos & $\mathrm{AC}$ & $\mathrm{AC}$ \\
\hline \multirow{3}{*}{ GRUPO 5} & Franca & $\mathrm{AC}$ & DEC \\
\hline & Limeira & $\mathrm{AC}$ & SIMÉTRICA \\
\hline & Piracicaba & $\mathrm{AC}$ & $\mathrm{AC}$ \\
\hline \multirow{3}{*}{ GRUPO 6} & Campinas & $\mathrm{AC}$ & DEC \\
\hline & Osasco & $\mathrm{AC}$ & $\mathrm{AC}$ \\
\hline & Sorocaba & $\mathrm{AC}$ & $\mathrm{AC}$ \\
\hline
\end{tabular}

Nota: AC - Transmissão mais intensa dos acréscimos; DEC - Transmissão mais intensa dos decréscimos; SIMÉTRICA - transmissão simétrica de acréscimos e decréscimos de preços.

Fonte: Resultados da pesquisa.

mais intensamente do que os decréscimos para todos os municípios, com exceção de Presidente Prudente.

Em relação ao efeito total, também houve predomínio de transmissão de acréscimos, mas o número de exceções foi maior. Dos 18 municípios, os acréscimos foram transmitidos mais intensamente para 11 (equivalente a 61,1\%), os decréscimos foram transmitidos mais intensamente para quatro $(22,2 \%)$ e houve três casos $(16,7 \%)$ de simetria.

Outro aspecto relevante é a rapidez da resposta. Em 15 dos 18 municípios estudados $(83,3 \%)$ os acréscimos já são transmitidos, totalmente, no mesmo mês, enquanto que os decréscimos são distribuídos em dois meses em todos os municípios.

Portanto, os resultados apresentam indicações de exercício de poder de mercado na maioria dos municípios, embora alguns deles só consigam transmitir mais intensamente os aumentos de preços no mês corrente, não conseguindo manter este efeito no mês subsequente. Verifica-se ainda que não existe um padrão claro indicando que o poder de mercado estaria mais presente nos municípios menores, contrariando a segunda hipótese que foi levantada neste trabalho. Porém, caso se compare os três grupos de municípios menores com os três grupos de municípios maiores, percebe-se que houve mais casos de transmissão de decréscimos de preços no grupo de municípios maiores (33,3\% dos casos) do que no grupo de municípios menores (11,1\% dos casos). Este fato pode, de certa forma, ser um indício de que em municípios menores haja maior exercício de poder de mercado devido a menor concorrência. Entretanto, novos estudos com mais municípios em cada grupo, precisariam ser feitos para se verificar mais acuradamente essa questão.

\section{Conclusões}

Os resultados do trabalho apresentam indicações de exercício de poder de mercado por parte dos postos de combustíveis na maioria dos municípios estudados. Mais especificamente, constatou-se que o exercício de poder de mercado está 
mais associado à rapidez com que os acréscimos de preços são transmitidos (apenas um município não transmitiu mais intensamente os acréscimos de preços no mês corrente) do que com a magnitude total do efeito. Mesmo assim, considerando o efeito total, em mais de $60 \%$ dos municípios os acréscimos de preços foram transmitidos mais intensamente tanto no curto quanto no longo prazo.

Os grupos de municípios construídos a partir do tamanho da população não apresentaram padrões muito definidos. Este fato pode ser decorrência do número reduzido de municípios incluído em cada grupo, ou de aspectos locais, impossíveis de serem identificados num estudo agregado como este, que poderiam estar afetando o poder de mercado das firmas e as formas de concorrência predominantes nos municípios mais do que o tamanho populacional. Ainda assim, mais casos de transmissão mais intensa de decréscimos de preços foram constatados nos municípios maiores, o que pode ser um indício de que nesses municípios o poder de mercado seja menor.

Dessa forma, os resultados encontrados no presente estudo trazem diversas implicações, tanto para o direcionamento de futuras pesquisas quanto para a implementação de políticas públicas dos órgãos de defesa da concorrência.

A utilização de testes de assimetria na transmissão de preços representa uma excelente alternativa para as análises do uso de poder de mercado, fornecendo resultados mais confiáveis do que as análises que se baseiam apenas em margens de comercialização ou em simples aumentos de preços, pois as firmas somente poderiam sistematicamente evitar ou retardar transmissões de quedas de preços caso desfrutassem de poder de mercado.

Ademais, a evidência de exercício de poder de mercado constatada neste trabalho indica que os órgãos de defesa da concorrência devem se manter atentos às eventuais fusões, sejam elas verticais ou horizontais, que afetem o nível de concentração do mercado varejista de etanol, assim como às condutas das empresas varejistas.

As limitações presentes neste trabalho estão diretamente relacionadas às características da base de dados disponível e ao método empregado. Como a base de dados fornece informações sobre os preços médios cobrados pela amostra de postos de cada município, não é possível identificar outras variáveis que podem originar poder de mercado, tais como o número de postos do mesmo município que pertencem aos mesmos grupos empresariais ou o histórico de concorrência de cada município. Outra limitação diz respeito ao tamanho do mercado. Como a base de dados só permite definir cada município como um mercado, o mercado relevante estará sendo superdimensionado no caso dos municípios maiores. Em termos metodológicos, a limitação decorre de se usar uma única variável, o preço, como parâmetro de análise, além do fato de se usar o procedimento de Houck, sendo que outras formas de análise da assimetria podem também ser empregadas. Entretanto, apesar dessas limitações, os resultados mostram fortes indícios de exercício do poder de mercado, justificando-se a preocupação que existe em relação ao abuso de poder de mercado no varejo de combustíveis.

Finalizando, sugere-se que novos estudos sejam feitos visando aumentar o conhecimento sobre o problema em apreço. Novos estudos poderiam incluir uma gama maior de municípios, quer do estado de São Paulo, quer de outro estado da federação, ou poderiam se concentrar em um único município (caso se obtivesse uma série de preços de cada posto de um município). Novos métodos de análise de assimetria também poderiam ser testados, para outras ou para as mesmas séries aqui utilizadas, visando identificar a dependência dos resultados em relação aos vários métodos de análise. Outro aspecto de grande relevância a ser examinado é a relação entre o preço do etanol e o preço da gasolina, uma vez que o preço desta pode limitar a transmissão de preços do etanol. 


\section{Referências}

AGUIAR, D. R. D. Mensuração de Poder de Mercado Por Meio de Dados de Marcas Comerciais: modelos, limitações e aplicações. Revista de Economia Política, v. 20, n. 3, p. 152-162, 2000.

. e FIGUEIREDO, A. M. Poder de mercado no varejo alimentar: uma análise usando os preços do estado de São Paulo. Revista de Economia e Sociologia Rural, v. 40, n. 04, p. 967-990, 2011.

. e SILVA, A. L. Changes in beef consumption and retailing competitiveness in Brazil: a rapid appraisal. Agribusiness, v. 18, n. 2, p. 145-161, 2002.

BARROS, G. S. A. C. Economia da Comercialização Agrícola. Piracicaba: FEALQ, 1987.

ANP. Levantamento de preços. Agência Nacional do Petróleo, Gás Natural e Biocombustíveis, 2011. Disponível em: <www.anp.gov.br>. Acesso em: 23 dez. 2011.

Anuário estatístico brasileiro do petróleo, gás natural e biocombustíveis 2013, Agência Nacional do Petróleo, Gás Natural e Biocombustíveis. Disponível em: <www.anp.gov.br>. Acesso em: 05 ago. 2013.

CARVALHO, L. H. e AGUIAR, D. R. D. Poder de monopsônio na indústria brasileira de esmagamento de soja. Revista de Economia e Agronegócio, Viçosa, v. 3, n. 3, p. 323-348, 2005.

CHURCH, J. e WARE, R. Industrial Organization: a Strategic Approach. San Francisco: McGraw-Hill, 2000.

COTTERILL, R. W., FRANKLIN, A. e MA, L. Y. Measuring Market Power Effects in Differentiated Product Industries: An Application to the Soft Drink Industry. Food Marketing Policy Center Research Report, \# 32 (April). Department of Agricultural and Resource Economics, University of Connecticut, 1996.

FIPE. 'Índice de preços ao consumidor', Fundação Instituto de Pesquisa Econômica, 2011. Disponível em: <www.fipe.org.br>. Acesso em: 06 set. 2011.

HAMILTON, J. D. Time series analysis. Princeton: Princeton University Press, 1994.

HOFFMANN, R. Análise de regressão - uma introdução à econometria. Campinas: Hucitec, 2006.

HOUCK, J. P. An approach to specifying and estimating nonreversible functions. American Journal of Agricultural Economics, v. 59, n. 3, p. 570-72, 1977.

IBGE. 'Censo Demográfico 2010', Instituto Brasileiro de Geografia e Estatística, 2011. Disponível em: < http:// www.ibge.gov.br/>. Acesso em: 20 jan. 2011.
JOHANSEN, S. Statistical analysis of cointegration vectors. Journal of Economic Dynamics and Control, v. 12, p. 231-254, 1988.

LASS, D. A. Asymmetric response of retail milk prices in the Northeast revisited. Agribusiness, v. 21, n. 4, p. 493-508, 2005.

LEVY,D., CHEN,H., RAY, S. eBERGEN,M. E. Asymmetric price adjustment in the small. Kiel Institute for World Economics. Kiel Working Paper No. 1356, 2007.

MARTIN, S. Industrial economics: economic analysis and public policy. 2. ed. Englewood Cliffs: Prentice Hall, 1993.

MEYER, J. e VON CRAMON-TAUBADEL, S. Asymmetric price transmission: a survey. Journal of Agricultural Economics, v. 55, n. 3, p. 581-611, 2004.

NG, S. e PERRON, P. Lag length selection and the construction of unit root tests with good size and power', Econometrica, v. 69, n. 6, p. 1519-1554, 2001.

PELTZMAN, S. Prices rise faster than they fall. Journal of Political Economy, v. 108, n. 31, p. 466-502, 2000.

RAY, S., CHEN, H., BERGEN, M. E. e LEVY, D. Asymmetric wholesale pricing: theory and evidence. Marketing Science, v. 25, n. 2, p. 131-154, 2006.

SDE.Combate a cartéis na revenda de combustíveis Brasil, Secretaria de Direito Econômico, Ministério da Justiça, 2009. Disponível em: <http://www.mp.sp.gov.br/portal/ page/portal/Cartilhas/CarteisRevendaCombustiveis. pdf > . Acesso em: 09 ago. 2013.

SILVA, C. C., MUNIZ, L. M., ARAUJO, N. G., ALMEIDA, R. S. e FIGUEIREDO, N. R. Investigando a assimetria e hysteresis nos preços dos combustíveis no mercado brasileiro: uma abordagem através dos modelos de THRESHOLD e ARFIMA'. In: CONGRESSO BRASILEIRO DE ECONOMIA E SOCIOLOGIA RURAL, 48, 2010, Campo Grande, MS. Anais... Brasília: Sociedade Brasileira de Economia e Sociologia Rural, 2010.

UCHÔA, A. F. A. Testando a assimetria nos preços da gasolina brasileira. Revista Brasileira de Economia, v. 62, n. 1, p. 103-117, 2008.

UNICA. Frota brasileira de autoveículos Leves (ciclo Otto). União da Indústria de Cana-de-Açúcar, 2013. Disponível em: <www.unica.com.br>. Acesso em: 07 out. 2013.

WARD, R. W. Asymmetry in retail, wholesale and shipping point pricing for fresh vegetables. American Journal of Agricultural Economics, v. 64, n. 2, p. 205-212, 1982.

WOLFRAM, R. Positivistic measures of aggregate supply elasticities: some new approaches- some critical notes. American Journal of Agricultural Economics, v. 53, n. 2, p. 356-359, 1971. 
